UCLA/99/22

June, 1999

\title{
Instanton Recursion Relations for the Effective Prepotential in $\mathcal{N}=2$ Super Yang-Mills $\mathbb{\theta}$
}

\author{
Gordon Chan], Eric D'Hoker] \\ Department of Physics and Astronomy \\ University of California \\ Los Angeles, CA 90095, USA
}

\begin{abstract}
Linear recursion relations for the instanton corrections to the effective prepotential of $\mathcal{N}=2$ supersymmetric gauge theories with an arbitrary number of hypermultiplets in the fundamental representation of an arbitrary classical gauge group are derived. The construction proceeds from the Seiberg-Witten solutions and the renormalization group type equations for the prepotential. Successive iterations of these recursion relations allow us to simply obtain instanton corrections to arbitrarily high order, which we exhibit explicitly up to 6-th order. For gauge groups $S U(2)$ and $S U(3)$, our results agree with previous ones.
\end{abstract}

\footnotetext{
${ }^{1}$ Research supported in part by the National Science Foundation under grants PHY9819686 and PHY-95-31023

${ }^{2}$ E-mail: morgoth@physics.ucla.edu

${ }^{3}$ E-mail: dhoker@physics.ucla.edu
} 


\section{Introduction}

Over the past few years there has been great progress in understanding non-perturbative dynamics of $\mathcal{N}=2$ SUSY gauge theories starting with the $\mathrm{SU}(2)$ case [1], further generalizations to other gauge groups [2] and additions of matter hypermultiplets [3]. Non-perturbative corrections in weak coupling correspond to instanton effects [4] which were evaluated using field theory techniques to one instanton [4] [5] [6] [7] and two instanton 8] orders.

Some of the previous instanton calculations using the Seiberg-Witten ansatz were performed by solving the Picard-Fuchs equations for the period integrals corresponding to the quantum moduli parameters representing the set of vacuum expectation values of the Higgs fields [9] [17]. Other previous calculations involved solving the period integrals directly [10], and were found to be in agreement with the Picard-Fuchs and field theory results. In an intriguing paper [11], a recursion relation for the instanton corrections to the effective prepotential $\mathcal{F}$ was found for the pure $S U(2)$ case which led us to seek a generalization of this result for any gauge group and number of matter hypermultiplets. In a related development, the Seiberg-Witten equations were viewed analogously to the Whitham hierarchy equations 12 and the WDVV equations [13. Nonlinear recursion relations for the instanton corrections involving Jacobi $\theta$-functions (which themselves involve $\tau_{i j}$ as in [16]) were derived starting from the Whitham hierarchy equations [14]. The beta function of the prepotential $\mathcal{F}$, first observed in 15 and later proved in [16], is the starting point of our discussion and provides a very direct way at calculating instanton corrections to the prepotential $\mathcal{F}$ without having to perform complicated hyperelliptic integrals and immediately obtaining rational expressions without $\theta$-functions.

The Seiberg-Witten solutions for classical gauge groups $S U(N), S O(N)$, and $S p(N)$ and the renormalization group like equation for the prepotential $\mathcal{F}$, led us to the discovery of a general recursion relation expressing the $n-t h$ order instanton correction to the prepotential $\mathcal{F}$ in terms of the $(n-$ 1)th, $\cdots, 1$ st order instanton corrections for super Yang-Mills theory with matter hypermultiplets in the fundamental representation of classical gauge groups $G$.

We start off by reviewing the Seiberg-Witten solution for $\mathcal{N}=2$ super Yang-Mills theory with hypermultiplets in the fundamental representation of any arbitrary classical gauge group. The renormalization group type equation 
for the prepotential $\mathcal{F}$ is discussed next and it is shown how it can be used to determine the instanton corrections to the prepotential to arbitrary order in an efficient manner. Recursion relations for the instanton corrections are then derived and shown to reproduce previous results.

\section{The Seiberg-Witten Solution for Arbitrary Classi- cal Gauge Group $G$}

The Seiberg-Witten (SW) ansatz gives a prescription for determining the prepotential of the effective action for $\mathcal{N}=2$ supersymmetric Yang-Mills gauge theories, as well as for determining the spectrum of BPS states.

We consider, $\mathcal{N}=2$ SUSY gauge theories with classical gauge groups $S U(r+1), S O(2 r+1), S p(2 r)$ and $S O(2 r)$, of rank $r$ and number of colours $N_{c}=r+1,2 r+1,2 r$, and $2 r$ respectively. We include $N_{f}$ hypermultiplets in the fundamental representation of the gauge group, with bare masses $m_{j}, j=1, \cdots, N_{f}$. We restrict to the asymptotically free theories; this limits the hypermultiplet contents $N_{f}$. (ie. $N_{f}<2 N_{c}$ for $S U\left(N_{c}\right)$ ). The classical vacuum expectation value of the gauge scalar $\phi$ is parameterized by complex moduli $\bar{a}_{k}, k=1, \cdots, r$ as follows

$$
\begin{aligned}
S U(r+1) & \phi & =\operatorname{diag}\left[\bar{a}_{1}, \cdots, \bar{a}_{r}, \bar{a}_{r+1}\right] & \bar{a}_{1}+\cdots+\bar{a}_{r}+\bar{a}_{r+1}=0 \\
S O(2 r+1) & \phi & =\operatorname{diag}\left[\mathcal{A}_{1}, \cdots, \mathcal{A}_{r}, 0\right] & \\
S p(2 r) & \phi & =\operatorname{diag}\left[\bar{a}_{1},-\bar{a}_{1}, \cdots, \bar{a}_{r},-\bar{a}_{r}\right] & \\
S O(2 r) & \phi & =\operatorname{diag}\left[\mathcal{A}_{1}, \cdots, \mathcal{A}_{r}\right] & \mathcal{A}_{k}=\left(\begin{array}{cc}
0 & \bar{a}_{k} \\
-\bar{a}_{k} & 0
\end{array}\right)
\end{aligned}
$$

For generic $\bar{a}_{k}$ 's, the gauge symmetry is broken to $U(1)^{r}$ and the dynamics is that of an Abelian Coulomb phase. The Wilson effective Lagrangian of the quantum theory to leading order in the low momentum expansion in the Abelian Coulomb phase is completely characterized by a complex analytic prepotential $\mathcal{F}(a)$.

The SW ansatz for determining the full prepotential $\mathcal{F}$ is based on a choice of a fibration of spectral curves over the space of vacua, and of a meromorphic 1-form $d \lambda$ on each of these curves. The renormalized order parameters $a_{k}$ of the theory, their duals $a_{D, k}$, and the prepotential $\mathcal{F}$ are 
given by

$$
2 \pi i a_{k}=\oint_{A_{k}} d \lambda, \quad 2 \pi i a_{D, k}=\oint_{B_{k}} d \lambda, \quad a_{D, k}=\frac{\partial \mathcal{F}}{\partial a_{k}}
$$

with $A_{k}, B_{k}$ a suitable set of homology cycles on the spectral curves [2].

For all $\mathcal{N}=2$ supersymmetric gauge theories based on classical gauge groups with $N_{f}$ hypermultiplets in the fundamental representation of the gauge group, the spectral curves and meromorphic 1-forms are

$$
\begin{array}{r}
y^{2}=A^{2}(x)-B(x) \\
d \lambda=\frac{x}{y}\left(A^{\prime}-\frac{A B^{\prime}}{2 B}\right) d x
\end{array}
$$

where

$$
\begin{array}{r}
S U(r+1) \quad A(x)=\prod_{k=1}^{r+1}\left(x-\bar{a}_{k}\right) \quad B(x)=\bar{\Lambda}^{2} \prod_{j=1}^{N_{f}}\left(x+m_{j}\right) \\
\left.\begin{array}{r}
S O(2 r+1) \\
S p(2 r)^{1} \\
S O(2 r)
\end{array}\right\} \quad A(x)=x^{a} \prod_{k=1}^{r}\left(x^{2}-\bar{a}_{k}^{2}\right) \quad B(x)=\bar{\Lambda}^{2} x^{b} \prod_{j=1}^{N_{f}}\left(x^{2}-m_{j}^{2}\right)
\end{array}
$$

with $\bar{\Lambda} \equiv \Lambda^{q}$

$$
\begin{array}{rlll}
S U(r+1) & q=r+1-N_{f} / 2 & & \\
S O(2 r+1) & q=2 r-1-N_{f} & a=0 & b=2 \\
S p(2 r) & q=2 r+2-N_{f} & a=2 & b=0 \\
S O(2 r) & q=2 r-2-N_{f} & a=0 & b=4
\end{array}
$$

respectively. The spectral curves (4) for $S O(2 r+1), S p(2 r)$ and $S O(2 r)$ can be obtained from the $S U(2 r)$ spectral curve by a suitable restriction on the classical moduli $\bar{a}_{k}$ 's and masses [19].

For gauge theories with classical gauge groups and asymptotically free coupling obeying the constraint $q>0$, general arguments based on the holomorphicity of $\mathcal{F}$, perturbative non-renormalization theorems beyond 1-loop

\footnotetext{
${ }^{1}$ For simplicity, we restrict attention here to the $\mathrm{Sp}(2 \mathrm{r})$ case with at least two massless hypermultiplets. The cases with one or no massless hypermultiplets may be treated accordingly 19$]$.
} 
order, the nature of instanton corrections, and restrictions of $U(1)_{R}$ invariance, constrain $\mathcal{F}$ to have the form

$$
\begin{aligned}
\mathcal{F}(a)= & \frac{2 q}{\pi i} \sum_{i=1}^{r} a_{i}^{2}+\frac{i}{4 \pi}\left[\sum_{\alpha}(\alpha \cdot a)^{2} \log \frac{(\alpha \cdot a)^{2}}{\Lambda^{2}}\right. \\
& \left.-\sum_{i} \sum_{j=1}^{N_{f}}\left(\lambda_{i} \cdot a+m_{j}\right)^{2} \log \frac{\left(\lambda_{i} \cdot a+m_{j}\right)^{2}}{\Lambda^{2}}\right] \\
+ & \sum_{m=1}^{\infty} \frac{\Lambda^{2 m q}}{2 m \pi i} \mathcal{F}^{(m)}(a)
\end{aligned}
$$

where $\lambda_{i}= \pm e_{i}$ for SO and Sp and $\lambda_{i}=e_{i}$ for SU in an orthonormal basis $\vec{e}_{i}$, and $\alpha$ are the roots of the gauge group $G$. (The $S U(r)$ solution requires an additional overall factor of $\frac{1}{2}$ ).

The terms on the right side are respectively the classical prepotential, the contribution of perturbative one-loop effects, and $m$-instanton processes contributions 2]. $\Lambda$ is the dynamically generated scale of the theory.

\section{Renormalization Group Type Equations}

In [16], a renormalization group type equation for the prepotential $\mathcal{F}$ was derived using the SW ansatz equations (2)

$$
\Lambda \frac{\partial \mathcal{F}}{\partial \Lambda}=\frac{q}{\pi i} \sum_{k=1}^{r} \bar{a}_{k}^{2}
$$

up to an additive term independent of $a_{k}$ and $\bar{a}_{k}$ which is physically immaterial. (The $S U(r)$ case requires an additional factor of $\frac{1}{2}$ ).

In [10] an efficient algorithm was presented for calculating the renormalized order parameters $a_{k}$ and their duals $a_{D, k}$ in terms of the classical order parameters $\bar{a}_{k}$ to any order of perturbation theory in a regime where $\bar{\Lambda}$ is small and the $\bar{a}_{k}$ 's are well-separated. The calculation of $a_{k}$ starts off from equations (2) and (3) producing a final result

$$
a_{k}=\sum_{m=0}^{\infty} \bar{\Lambda}^{2 m} \Delta_{k}^{(m)}(\bar{a})
$$

\footnotetext{
${ }^{2}$ The normalization of the instanton contributions in the present paper differs from that of [10] [19 by a factor $\frac{1}{4 m \pi i}$ for $S U\left(N_{c}\right)$ and $\frac{1}{2 m \pi i}$ for $S O(2 r+1), S p(2 r)$, and $S O(2 r)$. For our purposes, it will be convenient to use the normalization of (6).
} 
where we set $\Delta_{k}^{(0)}(\bar{a}) \equiv \bar{a}_{k}$, and we have

$$
\Delta_{k}^{(m)}(\bar{a})=\frac{1}{2^{2 m}(m !)^{2}}\left(\frac{\partial}{\partial \bar{a}_{k}}\right)^{2 m-1} S_{k}\left(\bar{a}_{k}, \bar{a}\right)^{m}, \quad m \neq 0
$$

with

$$
\begin{aligned}
S U(r+1) & S_{k}(x, a)=\frac{\prod_{j=1}^{N_{f}}\left(x+m_{j}\right)}{\prod_{l \neq k}\left(x-a_{l}\right)^{2}} \\
S O(2 r+1) & S_{k}(x, a)=\frac{x^{2} \prod_{j=1}^{N_{f}}\left(x^{2}-m_{j}^{2}\right)}{\left(x+a_{k}\right)^{2} \prod_{l \neq k}\left(x^{2}-a_{l}^{2}\right)^{2}} \\
S p(2 r)^{1} & S_{k}(x, a)=\frac{\prod_{j=1}^{N_{f}-2}\left(x^{2}-m_{j}^{2}\right)}{\left(x+a_{k}\right)^{2} \prod_{l \neq k}\left(x^{2}-a_{l}^{2}\right)^{2}} \\
S O(2 r) & S_{k}(x, a)=\frac{x^{4} \prod_{j=1}^{N_{f}}\left(x^{2}-m_{j}^{2}\right)}{\left(x+a_{k}\right)^{2} \prod_{l \neq k}\left(x^{2}-a_{l}^{2}\right)^{2}}
\end{aligned}
$$

and $\bar{\Lambda}$ defined as previously (5).

Equations (7) (8) (9) (10) suffices to determine the prepotential $\mathcal{F}$ in terms of the renormalized order parameters $a_{k}$ order by order in powers of $\bar{\Lambda}^{2}$.

\section{Recursion Relation for the Prepotential $\mathcal{F}$}

A very direct way of deriving the form of the instanton corrections to the prepotential $\mathcal{F}$ starts off from the beta function on the right hand side of (7). Substituting the ansatz for the prepotential (6) into the beta function (7)), one obtains

$$
\sum_{k=1}^{r} \bar{a}_{k}^{2}=\sum_{k=1}^{r} a_{k}^{2}+\sum_{m=1}^{\infty} \bar{\Lambda}^{2 m} \mathcal{F}^{(m)}(a)
$$

Substituting (8) into (11), one obtains

$$
\begin{aligned}
0= & \sum_{k=1}^{r}\left[\sum_{m=0}^{\infty} \bar{\Lambda}^{2 m} \Delta_{k}^{(m)}(\bar{a})\right]^{2}-\sum_{k=1}^{r}\left(\Delta_{k}^{(0)}(\bar{a})\right)^{2} \\
& +\sum_{m=1}^{\infty} \bar{\Lambda}^{2 m} \mathcal{F}^{(m)}\left(\sum_{n=0}^{\infty} \bar{\Lambda}^{2 n} \Delta_{k}^{(n)}(\bar{a})\right)
\end{aligned}
$$

Expanding in powers of $\bar{\Lambda}^{2}$ in the last term and replacing the $\bar{a}_{k}$ 's with $a_{k}$ 's, 
the $m$-th order instanton correction to the prepotential $\mathcal{F}$ takes on the form

$$
\begin{aligned}
-\mathcal{F}^{(m)}(a) & =\sum_{k=1}^{r}\left[\sum_{\substack{i, j=0 \\
i+j=m}}^{m} \Delta_{k}^{(i)}(a) \Delta_{k}^{(j)}(a)\right] \\
& +\sum_{n=1}^{m-1} \frac{1}{n !} \sum_{\substack{\beta_{1}, \cdots, \beta_{n+1}=1 \\
\beta_{1}+\cdots+\beta_{n+1}=m}}^{n-1} \sum_{\alpha_{1}, \cdots, \alpha_{n}=1}^{r}\left[\prod_{i=1}^{n} \Delta_{\alpha_{i}}^{\left(\beta_{i}\right)}(a)\right]\left(\prod_{j=1}^{n} \frac{\partial}{\partial a_{\alpha_{j}}}\right) \mathcal{F}^{\left(\beta_{n+1}\right)}(a)
\end{aligned}
$$

which is a linear recursion relation for $\mathcal{F}^{(m)}(a)$ in terms of the lower order instanton corrections $\mathcal{F}^{(m-1)}(a), \ldots, \mathcal{F}^{(1)}(a)$.

The intriguing part about the recursion relation (13) for $\mathcal{F}^{(n)}(a)$ is that it is linear in $\mathcal{F}^{(n-1)}(a), \cdots, \mathcal{F}^{(1)}(a)$ and is valid for all classical gauge groups with the number of hypermultiplets in the fundamental representation constrained by $q>0$. Previous recursion relations [11] were only valid for $S U(2)$ with no hypermultiplets and were non-linear.

\section{Instanton Expansion of the Prepotential $\mathcal{F}$}

Order by order in powers of $\bar{\Lambda}^{2}$, the first six instanton corrections (13) to the prepotential $\mathcal{F}$ are

$$
\begin{aligned}
-\mathcal{F}^{(1)}(a) & =\sum_{k=1}^{r} 2 \Delta_{k}^{(0)}(a) \Delta_{k}^{(1)}(a) \\
-\mathcal{F}^{(2)}(a) & =\sum_{k=1}^{r}\left[2 \Delta_{k}^{(0)}(a) \Delta_{k}^{(2)}(a)+\left(\Delta_{k}^{(1)}(a)\right)^{2}\right]+\sum_{k=1}^{r} \Delta_{k}^{(1)}(a) \frac{\partial \mathcal{F}^{(1)}(a)}{\partial a_{k}} \\
-\mathcal{F}^{(3)}(a) & =\sum_{k=1}^{r}\left[2 \Delta_{k}^{(0)}(a) \Delta_{k}^{(3)}(a)+2 \Delta_{k}^{(1)}(a) \Delta_{k}^{(2)}(a)\right] \\
& +\sum_{k=1}^{r}\left[\Delta_{k}^{(1)}(a) \frac{\partial \mathcal{F}^{(2)}(a)}{\partial a_{k}}+\Delta_{k}^{(2)}(a) \frac{\partial \mathcal{F}^{(1)}(a)}{\partial a_{k}}\right] \\
& +\frac{1}{2 !} \sum_{k, m=1}^{r} \Delta_{k}^{(1)}(a) \Delta_{m}^{(1)}(a) \frac{\partial^{2} \mathcal{F}^{(1)}(a)}{\partial a_{k} \partial a_{m}}
\end{aligned}
$$




$$
\begin{aligned}
& -\mathcal{F}^{(4)}(a)=\sum_{k=1}^{r}\left[2 \Delta_{k}^{(0)}(a) \Delta_{k}^{(4)}(a)+2 \Delta_{k}^{(1)}(a) \Delta_{k}^{(3)}(a)+\left(\Delta_{k}^{(2)}(a)\right)^{2}\right] \\
& +\sum_{k=1}^{r}\left[\Delta_{k}^{(1)}(a) \frac{\partial \mathcal{F}^{(3)}(a)}{\partial a_{k}}+\Delta_{k}^{(2)}(a) \frac{\partial \mathcal{F}^{(2)}(a)}{\partial a_{k}}+\Delta_{k}^{(3)}(a) \frac{\partial \mathcal{F}^{(1)}(a)}{\partial a_{k}}\right] \\
& +\frac{1}{2 !} \sum_{k, m=1}^{r}\left[\Delta_{k}^{(1)}(a) \Delta_{m}^{(1)}(a) \frac{\partial^{2} \mathcal{F}^{(2)}(a)}{\partial a_{k} \partial a_{m}}+2 \Delta_{k}^{(1)}(a) \Delta_{m}^{(2)}(a) \frac{\partial^{2} \mathcal{F}^{(1)}(a)}{\partial a_{k} \partial a_{m}}\right] \\
& +\frac{1}{3 !} \sum_{k, m, n=1}^{r} \Delta_{k}^{(1)}(a) \Delta_{m}^{(1)}(a) \Delta_{n}^{(1)}(a) \frac{\partial^{3} \mathcal{F}^{(1)}(a)}{\partial a_{k} \partial a_{m} \partial a_{n}} \\
& -\mathcal{F}^{(5)}(a)=\sum_{k=1}^{r}\left[2 \Delta_{k}^{(0)}(a) \Delta_{k}^{(5)}(a)+2 \Delta_{k}^{(1)}(a) \Delta_{k}^{(4)}(a)+2 \Delta_{k}^{(2)}(a) \Delta_{k}^{(3)}(a)\right] \\
& +\sum_{k=1}^{r}\left[\Delta_{k}^{(1)}(a) \frac{\partial \mathcal{F}^{(4)}(a)}{\partial a_{k}}+\Delta_{k}^{(2)}(a) \frac{\partial \mathcal{F}^{(3)}(a)}{\partial a_{k}}+\Delta_{k}^{(3)}(a) \frac{\partial \mathcal{F}^{(2)}(a)}{\partial a_{k}}\right. \\
& \left.+\Delta_{k}^{(4)}(a) \frac{\partial \mathcal{F}^{(1)}(a)}{\partial a_{k}}\right]+\frac{1}{2 !} \sum_{k, m=1}^{r}\left[\Delta_{k}^{(1)}(a) \Delta_{m}^{(1)}(a) \frac{\partial^{2} \mathcal{F}^{(3)}(a)}{\partial a_{k} \partial a_{m}}\right. \\
& +2 \Delta_{k}^{(1)}(a) \Delta_{m}^{(3)}(a) \frac{\partial^{2} \mathcal{F}^{(1)}(a)}{\partial a_{k} \partial a_{m}} \\
& \left.+\Delta_{k}^{(2)}(a) \Delta_{m}^{(2)}(a) \frac{\partial^{2} \mathcal{F}^{(1)}(a)}{\partial a_{k} \partial a_{m}}+2 \Delta_{k}^{(1)}(a) \Delta_{m}^{(2)}(a) \frac{\partial^{2} \mathcal{F}^{(2)}(a)}{\partial a_{k} \partial a_{m}}\right] \\
& +\frac{1}{3 !} \sum_{k, m, n=1}^{r}\left[\Delta_{k}^{(1)}(a) \Delta_{m}^{(1)}(a) \Delta_{n}^{(1)}(a) \frac{\partial^{3} \mathcal{F}^{(2)}(a)}{\partial a_{k} \partial a_{m} \partial a_{n}}\right. \\
& \left.+3 \Delta_{k}^{(2)}(a) \Delta_{m}^{(1)}(a) \Delta_{n}^{(1)}(a) \frac{\partial^{3} \mathcal{F}^{(1)}(a)}{\partial a_{k} \partial a_{m} \partial a_{n}}\right] \\
& +\frac{1}{4 !} \sum_{k, m, n, l=1}^{r} \Delta_{k}^{(1)}(a) \Delta_{m}^{(1)}(a) \Delta_{n}^{(1)}(a) \Delta_{l}^{(1)}(a) \frac{\partial^{4} \mathcal{F}^{(2)}(a)}{\partial a_{k} \partial a_{m} \partial a_{n} \partial a_{l}} \\
& -\mathcal{F}^{(6)}(a)=\sum_{k=1}^{r}\left[2 \Delta_{k}^{(0)}(a) \Delta_{k}^{(6)}(a)+2 \Delta_{k}^{(1)}(a) \Delta_{k}^{(5)}(a)+2 \Delta_{k}^{(2)}(a) \Delta_{k}^{(4)}(a)\right. \\
& \left.+\left(\Delta_{k}^{(3)}(a)\right)^{2}\right]+\sum_{k=1}^{r}\left[\Delta_{k}^{(1)}(a) \frac{\partial \mathcal{F}^{(5)}(a)}{\partial a_{k}}+\Delta_{k}^{(2)}(a) \frac{\partial \mathcal{F}^{(4)}(a)}{\partial a_{k}}\right. \\
& \left.+\Delta_{k}^{(3)}(a) \frac{\partial \mathcal{F}^{(3)}(a)}{\partial a_{k}}+\Delta_{k}^{(4)}(a) \frac{\partial \mathcal{F}^{(2)}(a)}{\partial a_{k}}+\Delta_{k}^{(5)}(a) \frac{\partial \mathcal{F}^{(1)}(a)}{\partial a_{k}}\right]
\end{aligned}
$$




$$
\begin{aligned}
& +\frac{1}{2 !} \sum_{k, m=1}^{r}\left[\Delta_{k}^{(1)}(a) \Delta_{m}^{(1)}(a) \frac{\partial^{2} \mathcal{F}^{(4)}(a)}{\partial a_{k} \partial a_{m}}+2 \Delta_{k}^{(1)}(a) \Delta_{m}^{(4)}(a) \frac{\partial^{2} \mathcal{F}^{(1)}(a)}{\partial a_{k} \partial a_{m}}\right. \\
& +\Delta_{k}^{(2)}(a) \Delta_{m}^{(2)}(a) \frac{\partial^{2} \mathcal{F}^{(2)}(a)}{\partial a_{k} \partial a_{m}}+2 \Delta_{k}^{(1)}(a) \Delta_{m}^{(2)}(a) \frac{\partial^{2} \mathcal{F}^{(3)}(a)}{\partial a_{k} \partial a_{m}} \\
& \left.+2 \Delta_{k}^{(1)}(a) \Delta_{m}^{(3)}(a) \frac{\partial^{2} \mathcal{F}^{(2)}(a)}{\partial a_{k} \partial a_{m}}+2 \Delta_{k}^{(2)}(a) \Delta_{m}^{(3)}(a) \frac{\partial^{2} \mathcal{F}^{(1)}(a)}{\partial a_{k} \partial a_{m}}\right] \\
& +\frac{1}{3 !} \sum_{k, m, n=1}^{r}\left[\Delta_{k}^{(1)}(a) \Delta_{m}^{(1)}(a) \Delta_{n}^{(1)}(a) \frac{\partial^{3} \mathcal{F}^{(3)}(a)}{\partial a_{k} \partial a_{m} \partial a_{n}}\right. \\
& +3 \Delta_{k}^{(1)}(a) \Delta_{m}^{(1)}(a) \Delta_{n}^{(3)}(a) \frac{\partial^{3} \mathcal{F}^{(1)}(a)}{\partial a_{k} \partial a_{m} \partial a_{n}} \\
& +3 \Delta_{k}^{(1)}(a) \Delta_{m}^{(1)}(a) \Delta_{n}^{(2)}(a) \frac{\partial^{3} \mathcal{F}^{(2)}(a)}{\partial a_{k} \partial a_{m} \partial a_{n}} \\
& \left.+3 \Delta_{k}^{(1)}(a) \Delta_{m}^{(2)}(a) \Delta_{n}^{(2)}(a) \frac{\partial^{3} \mathcal{F}^{(1)}(a)}{\partial a_{k} \partial a_{m} \partial a_{n}}\right] \\
& +\frac{1}{4 !} \sum_{k, m, n, l=1}^{r}\left[\Delta_{k}^{(1)}(a) \Delta_{m}^{(1)}(a) \Delta_{n}^{(1)}(a) \Delta_{l}^{(1)}(a) \frac{\partial^{4} \mathcal{F}^{(2)}(a)}{\partial a_{k} \partial a_{m} \partial a_{n} \partial a_{l}}\right. \\
& \left.+4 \Delta_{k}^{(2)}(a) \Delta_{m}^{(1)}(a) \Delta_{n}^{(1)}(a) \Delta_{l}^{(1)}(a) \frac{\partial^{4} \mathcal{F}^{(1)}(a)}{\partial a_{k} \partial a_{m} \partial a_{n} \partial a_{l}}\right] \\
& +\frac{1}{5 !} \sum_{k, m, n, l, o=1}^{r} \Delta_{k}^{(1)}(a) \Delta_{m}^{(1)}(a) \Delta_{n}^{(1)}(a) \Delta_{l}^{(1)}(a) \Delta_{o}^{(1)}(a) \frac{\partial^{5} \mathcal{F}^{(1)}(a)}{\partial a_{k} \partial a_{m} \partial a_{n} \partial a_{l} \partial a_{o}}
\end{aligned}
$$

A closer examination of the recursion relation (13) for the prepotential $\mathcal{F}$ reveals that there is always a term of the form

$$
2 \sum_{k=1}^{r} \Delta_{k}^{(0)}(a) \Delta_{k}^{(n)}(a)=2 \sum_{k=1}^{r} a_{k} \Delta_{k}^{(n)}(a)
$$

When performing explicit calculations for special cases of $N_{c}$ and $N_{f}$, it is useful to rewrite terms of the form (20) so that there are no $a_{k}$ 's sitting out in front. Using the definition (10) of $S_{k}(x, a)$ and performing contour integrals in the complex plane by residue methods as in [10], it can be shown that

$$
2 \sum_{k=1}^{r} a_{k} \Delta_{k}^{(n)}(a)=-\frac{(2 n-1)}{2^{2 n-1}(n !)^{2}} \sum_{k=1}^{r}\left(\frac{\partial}{\partial a_{k}}\right)^{2 n-2} S_{k}\left(a_{k}, a\right)^{n}
$$


up to an $a_{k}$ independent term that is physically immaterial for $q>0$.

\section{Comparison with Previous Results}

In order to make explicit comparisons with results in the literature, the instanton corrections have to be rewritten in terms of symmetric polynomials in the $a_{k}$ 's as follows.

For $S U(2)$, the existing results in the literature have the instanton expressions expressed in terms of

$$
\begin{aligned}
& a_{1}=2 a \\
& a_{2}=-2 a
\end{aligned}
$$

Solving the recursion relation (13) for the pure $S U(2)$ case, the explicit form for the n-th order instanton correction to the prepotential $\mathcal{F}$ was determined to be

$$
\mathcal{F}^{(n)}(a)=\frac{1}{(2 a)^{4 n-2}} \sum_{j=1}^{n}\left(\begin{array}{c}
4 n-3 \\
j-1
\end{array}\right) \frac{(-1)^{j-1}}{j} \sum_{\substack{n_{1}, \cdots, n_{j}=1 \\
n_{1}+\cdots+n_{j}=n}}^{n} b_{n_{1}} \cdots b_{n_{j}}
$$

where

$$
b_{n}=\frac{(2 n-3) ! !}{(n !)^{2}}
$$

which agrees with previous results [9] [10] अ

Explicit evaluations for $N_{f}=0,1,2,3$ were performed, with $N_{f}=3$ summarized here.

$$
\begin{aligned}
& \mathcal{F}^{(1)}=\frac{1}{2^{2} a^{2}}\left[a^{2}\left(m_{1}+m_{2}+m_{3}\right)+m_{1} m_{2} m_{3}\right] \\
& \mathcal{F}^{(2)}=\frac{1}{2^{8} a^{6}}\left[a^{6}+a^{4}\left(m_{1}^{2}+m_{2}^{2}+m_{3}^{2}\right)-a^{2}\left(m_{1}^{2} m_{2}^{2}+m_{1}^{2} m_{3}^{2}+m_{2}^{2} m_{3}^{2}\right)+5 m_{1}^{2} m_{2}^{2} m_{3}^{2}\right] \\
& \mathcal{F}^{(3)}=\frac{m_{1} m_{2} m_{3}}{2^{11} a^{10}}\left[-3 a^{6}+5 a^{4}\left(m_{1}^{2}+m_{2}^{2}+m_{3}^{2}\right)-7 a^{2}\left(m_{1}^{2} m_{2}^{2}+m_{1}^{2} m_{3}^{2}+m_{2}^{2} m_{3}^{2}\right)\right.
\end{aligned}
$$

\footnotetext{
${ }^{3}$ Our results agree exactly with those of [9] to eight instantons with the replacement $\Lambda^{2} \rightarrow \frac{\Lambda^{2}}{2}$.
} 


$$
\begin{aligned}
& \left.+\quad 9 m_{1}^{2} m_{2}^{2} m_{3}^{2}\right] \\
& \mathcal{F}^{(4)}=\frac{1}{2^{20} a^{14}}\left\{a^{12}-6 a^{10}\left(m_{1}^{2}+m_{2}^{2}+m_{3}^{2}\right)+a^{8}\left[5\left(m_{1}^{4}+m_{2}^{4}+m_{3}^{4}\right)\right.\right. \\
& \left.+100\left(m_{1}^{2} m_{2}^{2}+m_{1}^{2} m_{3}^{2}+m_{2}^{2} m_{3}^{2}\right)\right]+a^{6}\left[1176 m_{1}^{2} m_{2}^{2} m_{3}^{2}\right. \\
& \left.-126\left(m_{1}^{4} m_{2}^{2}+m_{1}^{2} m_{2}^{4}+m_{1}^{4} m_{3}^{2}+m_{1}^{2} m_{3}^{4}+m_{2}^{4} m_{3}^{2}+m_{2}^{2} m_{3}^{4}\right)\right] \\
& +a^{4}\left[153\left(m_{1}^{4} m_{2}^{4}+m_{1}^{4} m_{3}^{4}+m_{2}^{4} m_{3}^{4}\right)+1332 m_{1}^{2} m_{2}^{2} m_{3}^{2}\left(m_{1}^{2}+m_{2}^{2}+m_{3}^{2}\right)\right] \\
& \text { - } \left.1430 a^{2} m_{1}^{2} m_{2}^{2} m_{3}^{2}\left(m_{1}^{2} m_{2}^{2}+m_{1}^{2} m_{3}^{2}+m_{2}^{2} m_{3}^{2}\right)+1469 m_{1}^{4} m_{2}^{4} m_{3}^{4}\right\} \\
& \mathcal{F}^{(5)}=\frac{m_{1} m_{2} m_{3}}{2^{23} a^{18}}\left\{35 a^{12}-210 a^{10}\left(m_{1}^{2}+m_{2}^{2}+m_{3}^{2}\right)\right. \\
& +a^{8}\left[207\left(m_{1}^{4}+m_{2}^{4}+m_{3}^{2}\right)+1260\left(m_{1}^{2} m_{2}^{2}+m_{1}^{2} m_{3}^{3}+m_{2}^{2} m_{3}^{2}\right)\right] \\
& -1210 a^{6}\left(m_{1}^{4} m_{2}^{2}+m_{1}^{2} m_{2}^{4}+m_{1}^{4} m_{3}^{2}+m_{1}^{2} m_{3}^{4}+m_{2}^{4} m_{3}^{2}+m_{2}^{2} m_{3}^{4}\right) \\
& +a^{4}\left[1131\left(m_{1}^{4} m_{2}^{4}+m_{1}^{4} m_{3}^{4}+m_{2}^{4} m_{3}^{4}\right)+5960 m_{1}^{2} m_{2}^{2} m_{3}^{2}\left(m_{1}^{2}+m_{2}^{2}+m_{3}^{2}\right)\right] \\
& \text { - } \left.5250 a^{2} m_{1}^{2} m_{2}^{2} m_{3}^{2}\left(m_{1}^{2} m_{2}^{2}+m_{1}^{2} m_{3}^{2}+m_{2}^{2} m_{3}^{2}\right)+4471 m_{1}^{4} m_{2}^{4} m_{3}^{4}\right\} \\
& \mathcal{F}^{(6)}=\frac{1}{2^{29} a^{22}}\left\{5 a^{16}\left(m_{1}^{2}+m_{2}^{2}+m_{3}^{2}\right)-a^{14}\left[210\left(m_{1}^{2} m_{2}^{2}+m_{1}^{2} m_{3}^{2}+m_{2}^{2} m_{3}^{2}\right)\right.\right. \\
& \left.+14\left(m_{1}^{4}+m_{2}^{4}+m_{3}^{4}\right)\right]+a^{12}\left[9\left(m_{1}^{6}+m_{2}^{6}+m_{3}^{6}\right)+6507 m_{1}^{2} m_{2}^{2} m_{3}^{2}\right. \\
& \left.+801\left(m_{1}^{4} m_{2}^{2}+m_{1}^{2} m_{2}^{4}+m_{1}^{4} m_{3}^{2}+m_{1}^{2} m_{3}^{4}+m_{2}^{4} m_{3}^{2}+m_{2}^{2} m_{3}^{4}\right)\right] \\
& \text { - } a^{10}\left[660\left(m_{1}^{6} m_{2}^{2}+m_{1}^{2} m_{2}^{6}+m_{1}^{6} m_{3}^{2}+m_{1}^{2} m_{3}^{6}+m_{2}^{6} m_{3}^{2}+m_{2}^{2} m_{3}^{6}\right)\right. \\
& \left.+330\left(m_{1}^{4} m_{2}^{4}+m_{1}^{4} m_{3}^{4}+m_{2}^{4} m_{3}^{4}\right)+24420 m_{1}^{2} m_{2}^{2} m_{3}^{2}\left(m_{1}^{2}+m_{2}^{2}+m_{3}^{2}\right)\right] \\
& +a^{8}\left[2769\left(m_{1}^{6} m_{2}^{4}+m_{1}^{4} m_{2}^{6}+m_{1}^{6} m_{3}^{4}+m_{1}^{4} m_{3}^{6}+m_{2}^{6} m_{3}^{4}+m_{2}^{4} m_{3}^{6}\right)\right. \\
& \left.+m_{1}^{2} m_{2}^{2} m_{3}^{2}\left(19851\left(m_{1}^{4}+m_{2}^{4}+m_{3}^{4}\right)+87945\left(m_{1}^{2} m_{2}^{2}+m_{1}^{2} m_{3}^{2}+m_{2}^{2} m_{3}^{2}\right)\right)\right] \\
& \text { - } a^{6}\left[295050 m_{1}^{4} m_{2}^{4} m_{3}^{4}+2310\left(m_{1}^{2} m_{2}^{2}+m_{1}^{2} m_{3}^{2}+m_{2}^{2} m_{3}^{2}\right)\right. \\
& \left.+69510 m_{1}^{2} m_{2}^{2} m_{3}^{2}\left(m_{1}^{4} m_{2}^{2}+m_{1}^{2} m_{2}^{4}+m_{1}^{4} m_{3}^{2}+m_{1}^{2} m_{3}^{4}+m_{2}^{4} m_{3}^{2}+m_{2}^{2} m_{3}^{4}\right)\right] \\
& +a^{4} m_{1}^{2} m_{2}^{2} m_{3}^{2}\left[53839\left(m_{1}^{4} m_{2}^{4}+m_{1}^{4} m_{3}^{4}+m_{2}^{4} m_{3}^{4}\right)+224485 m_{1}^{2} m_{2}^{2} m_{3}^{2}\left(m_{1}^{2}+m_{2}^{2}+m_{3}^{2}\right)\right] \\
& \text { - } \left.166896 a^{2} m_{1}^{4} m_{2}^{4} m_{3}^{4}\left(m_{1}^{2} m_{2}^{2}+m_{1}^{2} m_{3}^{2}+m_{2}^{2} m_{3}^{2}\right)+121191 m_{1}^{6} m_{2}^{6} m_{3}^{6}\right\}
\end{aligned}
$$

A check of the hypermultiplet decoupling limits of the $N_{f}=3$ instanton corrections, by letting $\Lambda_{3} m_{3}=\Lambda_{2}^{2}$ and sending $m_{3} \rightarrow \infty$, reproduces the $N_{f}=2$ results. A further decoupling of a second hypermultiplet, by letting $\Lambda_{2} m_{2}=\Lambda_{1}^{2}$ and sending $m_{c} \rightarrow \infty$, reproduces the $N_{f}=1$ results. Comparison with results in the literature [17] [10] 14] show an agreement to four instantons up to a redefinition of the $\bar{a}_{k}$ 's as discussed in [10]. 
For $S U(3)$, the existing results in the literature have the instanton corrections expressed in terms of the invariant $S U(3)$ symmetric polynomials $u, v$ and the discriminant $\Delta$

$$
\begin{aligned}
u & =-a_{1} a_{2}-a_{1} a_{3}-a_{2} a_{3} \\
v & =a_{1} a_{2} a_{3} \\
\Delta & =4 u^{3}-27 v^{3}
\end{aligned}
$$

and the $p$-th symmetric mass polynomials

$$
t_{p}(m)=\sum_{j_{1}<\cdots<j_{p}} m_{j_{1}} \cdots m_{j_{p}}
$$

Explicit evaluations for $N_{f}=0,1,2,3,4,5$ were performed, and are summarized here for $N_{f}=0$.

$$
\begin{aligned}
\mathcal{F}^{(1)}= & \Lambda^{6} \frac{3 u}{\Delta} \\
\mathcal{F}^{(2)=} & \frac{\Lambda^{12} u}{16}\left[\frac{10935 v^{2}}{\Delta^{3}}+\frac{153}{\Delta^{2}}\right] \\
\mathcal{F}^{(3)=} & \frac{3 \Lambda^{18} u}{16}\left[\frac{4782969 v^{4}}{2 \Delta^{5}}+\frac{161109 v^{2}}{2 \Delta^{4}}+\frac{385}{\Delta^{3}}\right] \\
\mathcal{F}^{(4)}= & \frac{\Lambda^{24} u}{4096}\left[\frac{1707362095023 v^{6}}{\Delta^{7}}+\frac{91216001799 v^{4}}{\Delta^{6}}+\frac{1254600981 v^{2}}{\Delta^{5}}\right. \\
& +\frac{3048885}{\left.\Delta^{4}\right]}+\frac{5 \Lambda^{30} u}{4096}\left[\frac{3788227372819653 v^{8}}{10 \Delta^{9}}+\frac{277223767370307 v^{6}}{10 \Delta^{8}}\right. \\
& +\frac{6447389599341 v^{4}}{\left.10 \Delta^{7}+\frac{50110037721 v^{2}}{10 \Delta^{6}}+\frac{7400133}{\Delta^{5}}\right]} \\
\mathcal{F}^{(5)}= & \frac{3 \Lambda^{36} u}{65535}\left[\frac{24952152189682606959 v^{10}}{2 \Delta^{11}}+\frac{2319087386959542567 v^{8}}{2 \Delta^{10}}\right. \\
& +\frac{38185135433846901 v^{6}}{\Delta^{9}}+\frac{525166021552761 v^{4}}{\Delta^{8}} \\
\mathcal{F}^{(6)} & \left.+\frac{5323867298775 v^{2}}{2 \Delta^{7}}+\frac{5295230391}{2 \Delta^{6}}\right]
\end{aligned}
$$


A check of successive hypermultiplet decoupling limits of the $N_{f}=5$ instanton corrections reproduces all of the $N_{f}<5$ cases accordingly. Comparison with results in the literature [9] 10] 18] [14] show an agreement to three instantons up to a redefinition of the $\bar{a}_{k}$ 's as discussed in 10 .

\section{Summary}

The recursion relations discovered in this paper improve considerably the ability to evaluate explicitly the non-perturbative instanton corrections to

$\mathcal{N}=2$ super Yang-Mills theories. Possible extensions to other problems like the strongly coupled $\mathcal{N}=2$ SUSY $S U\left(N_{c}\right)$ Seiberg-Witten problem [20] 21] were also investigated [22].

\section{Acknowledgments}

We are grateful to D.H. Phong for several helpful discussions and collaboration at the very early stage of this work. G.C. would like to thank NSERC for financial support.

\section{Appendix}

\section{A. Classical Moduli in Terms of Quantum Moduli}

Another way of evaluating the beta function (7) of the prepotential $\mathcal{F}$ involves inverting (8) to get

$$
\bar{a}_{k} \equiv a_{k}+\sum_{m=1}^{\infty} \bar{\Lambda}^{2 m} \beta_{k}^{(m)}(a)
$$

where the $\beta_{k}(a)$ 's are functions of the renormalized order parameters $a_{k}$.

A very direct way of deriving the form of the $\beta_{k}(a)$ 's involves starting off with (27) and substituting in equation (8) to get

$$
0=\sum_{m=1}^{\infty} \bar{\Lambda}^{2 m} \Delta_{i}^{(m)}(\bar{a})+\sum_{m=1}^{\infty} \bar{\Lambda}^{2 m} \beta_{i}^{(m)}\left(\sum_{m=0}^{\infty} \bar{\Lambda}^{2 m} \Delta_{k}^{(m)}(\bar{a})\right)
$$


Expanding in powers of $\bar{\Lambda}^{2}$ in the second term and replacing the $\bar{a}_{k}$ 's with $a_{k}$ 's, one obtains

$$
\begin{aligned}
-\beta_{k}^{(m)}(a) & =\Delta_{k}^{(m)}(a) \\
& +\sum_{n=1}^{m-1} \frac{1}{n !} \sum_{\substack{\beta_{1}, \cdots, \beta_{n+1}=1 \\
\beta_{1}+\cdots+\beta_{n+1}=m}}^{n-1} \sum_{\alpha_{1}, \cdots, \alpha_{n}=1}^{r}\left[\prod_{i=1}^{n} \Delta_{\alpha_{i}}^{\left(\beta_{i}\right)}(a)\right]\left(\prod_{j=1}^{n} \frac{\partial}{\partial a_{\alpha_{j}}}\right) \beta_{k}^{\left(\beta_{n+1}\right)}(a)
\end{aligned}
$$

Order by order in powers of $\bar{\Lambda}^{2}$, the first few $\beta_{k}(a)$ 's are

$$
\begin{aligned}
-\beta_{k}^{(1)}(a) & =\Delta_{k}^{(1)}(a) \\
-\beta_{k}^{(2)}(a) & =\Delta_{k}^{(2)}(a)+\sum_{l=1}^{r} \Delta_{l}^{(1)}(a) \frac{\partial \beta_{k}^{(1)}(a)}{\partial a_{l}} \\
-\beta_{k}^{(3)}(a) & =\Delta_{k}^{(3)}(a)+\sum_{l=1}^{r}\left[\Delta_{l}^{(1)}(a) \frac{\partial \beta_{k}^{(2)}(a)}{\partial a_{l}}+\Delta_{l}^{(2)}(a) \frac{\partial \beta_{k}^{(1)}(a)}{\partial a_{l}}\right] \\
& +\frac{1}{2 !} \sum_{l, m=1}^{r} \Delta_{l}^{(1)}(a) \Delta_{m}^{(1)}(a) \frac{\partial^{2} \beta_{k}^{(1)}(a)}{\partial a_{l} \partial a_{m}} \\
-\beta_{k}^{(4)}(a) & =\Delta_{k}^{(4)}(a)+\sum_{l=1}^{r}\left[\Delta_{l}^{(1)}(a) \frac{\partial \beta_{k}^{(3)}(a)}{\partial a_{l}}+\Delta_{l}^{(2)}(a) \frac{\partial \beta_{k}^{(2)}(a)}{\partial a_{l}}\right. \\
& \left.+\Delta_{l}^{(3)}(a) \frac{\partial \beta_{k}^{(1)}(a)}{\partial a_{l}}\right]+\frac{1}{2 !} \sum_{l, m=1}^{r}\left[\Delta_{l}^{(1)}(a) \Delta_{m}^{(1)}(a) \frac{\partial^{2} \beta_{k}^{(2)}(a)}{\partial a_{l} \partial a_{m}}\right. \\
& \left.+2 \Delta_{l}^{(1)}(a) \Delta_{m}^{(2)}(a) \frac{\partial^{2} \beta_{k}^{(1)}(a)}{\partial a_{l} \partial a_{m}}\right] \\
& +\frac{1}{3 !} \sum_{l, m, n=1}^{r} \Delta_{l}^{(1)}(a) \Delta_{m}^{(1)}(a) \Delta_{n}^{(1)}(a) \frac{\partial^{3} \beta_{k}^{(1)}(a)}{\partial a_{l} \partial a_{m} \partial a_{n}}
\end{aligned}
$$

Substituting (29) into (11) reproduces the instanton corrections to the prepotential (13) order by order in $\bar{\Lambda}^{2}$. 


\section{References}

[1] N. Seiberg and E. Witten, "Electric-Magnetic Duality, Monopole Condensation, and Confinement in $\mathcal{N}=2$ Supersymmetric Yang-Mills Theory", Nucl. Phys. B 426 (1994) 19, hep-th/9407087;

N. Seiberg and E. Witten, "Monopoles, Duality and Chiral Symmetry Breaking in $\mathcal{N}=2$ Supersymmetric QCD”, Nucl. Phys. B 431 (1994) 484, hep-th/9408099.

[2] A. Klemm, W. Lerche, S. Yankielowicz, and S. Theisen, "Simple Singularities and $\mathcal{N}=2$ Supersymmetric Yang-Mills Theory", Phys. Lett. B 344 (1995) 169, hep-th/9411048;

P.C. Argyres and A. Faraggi, The Vacuum Structure and Spectrum of $\mathcal{N}=2$ Supersymmetric $S U(N)$ Gauge Theory, Phys. Rev. Lett. 73 (1995) 3931, hep-th/9411057;

J. Minahan and D. Nemeshansky, "Hyperelliptic Curves for Supersymmetric Yang-Mills", Nucl. Phys. B 464 (1996) 3, hep-th/9507032;

U.H. Danielsson and B. Sundborg, "The Moduli Space and Monodromies of $\mathcal{N}=2$ Supersymmetric $S O(2 R+1)$ Yang-Mills Theory", Phys. Lett. B 358 (1995) 273;

A. Brandhuber and K. Landsteiner, "On the Monodromies of $\mathcal{N}=2$ Supersymmetric Yang-Mills Theory with Gauge Group $S O(2 N)$, Phys. Lett. B 358 (1995) 73, hep-th/9507008;

M. Alishahiha, F. Ardalan and F. Mansouri, "The Moduli Space of the Supersymmetric G(2) Yang-Mills Theory", Phys. Lett. B 381 (1996) 446, hep-th/9512005;

E. Martinec and N. Warner, "Integrable Systems and Supersymmetric Gauge Theories", Nucl. Phys. B 459 (1996) 97.

[3] A. Hanany, Y. Oz, "On The Quantum Moduli Space of Vacua of $\mathcal{N}=2$ Supersymmetric $S U\left(N_{c}\right)$ Gauge Theories", Nucl. Phys. B 452 (1995) 73, hep-th/9505075;

A. Hanany, "On the Quantum Moduli Space of Vacua $\mathcal{N}=2$ Supersymmetric Gauge Theories", Nucl. Phys. B 466 (1996) 85, hep-th/9509176; P.C. Argyres, M.R. Plesser and A.D. Shapere, "The Coulomb Phase of $\mathcal{N}=2$ Supersymmetric QCD", Phys. Rev. Lett 75 (1995) 1699, hepth/9505100; 
P.C. Argyres and A. Shapere, "The Vacuum Structure of $\mathcal{N}=2$ SuperQCD with Classical Gauge Groups", Nucl. Phys. B 461 (1996) 437, hep-th/9509175;

D.H. Phong, I.M. Krichever, "On the Integrable Geometry of Soliton Equations and $\mathcal{N}=2$ Supersymmetric Gauge Theories", J. Diff. Geom. 45 (1997) 349, hep-th/9604199.

[4] N. Seiberg, "Supersymmetry and Nonperturbative Beta Functions", Phys. Lett. B 206 (1988) 75.

[5] V.A. Novikov, M.A. Shifman, A.I. Vainshtein and V.I. Zakharov, "Exact Gell-Mann-Low Function of Supersymmetric Yang-Mills Theories from Instanton Calculus", Nucl. Phys. B 229 (1983) 381.

[6] D. Finnell and P. Pouliot, "Instanton Calculations Versus Exact Results in Four-Dimensionsal SUSY Gauge Theories", Nucl. Phys. B 453 (1995) 225, hep-th/9503115.

[7] F. Fucito and G. Travaglini, "Instanton Calculus and Nonperturbative Relations in $\mathcal{N}=2$ Supersymmetric Gauge Theories", Phys. Rev. D 155 (1997) 1099, hep-th/9605215.

[8] N. Dorey, V. Khoze, and M.P. Mattis, "Multi-Instanton Calculus in $\mathcal{N}=2$ Supersymmetric Gauge Theory", Phys. Rev. D (1996) 2921, hepth/9603136;

N. Dorey, V. Khoze, and M.P. Mattis, "A Two Instanton Test of the Exact Solution of $\mathcal{N}=2$ Supersymmetric QCD”, Phys. Lett. B 388 (1996), 324, hep-th/9607066.

[9] A. Klemm, W. Lerche and S. Theisen, "Non-Perturbative Effective Actions of $\mathcal{N}=2$ Supersymmetric Gauge Theories", Int. J. Mod. Phys. A 11 (1996) 1929, hep-th/9505150.

[10] E. D'Hoker, D.H. Phong, I.M. Krichever, "The Effective Prepotential of $\mathcal{N}=2$ Supersymmetric $S U\left(N_{c}\right)$ Gauge Theories", Nucl. Phys. B 489 (1997) 179, hep-th/9609041.

[11] M. Matone, "Instantons and Recursion Relations in $\mathcal{N}=2$ SUSY Gauge Theory", Phys. Lett. B 357 (1995) 342, hep-th/9506102. 
[12] A. Gorsky, I.M. Krichever, A. Marshakov, A. Mironov and A. Morozov, "Integrability and Seiberg-Witten Exact Solution", Phys. Lett. B 355 (1995), hep-th/9505035;

H. Itoyama and A. Morozov, "Prepotential and the Seiberg-Witten Theory", Nucl. Phys. B 491 (1997) 529, hep-th/9512161;

T. Eguchi and S. Yang, "Prepotentials of $\mathcal{N}=2$ Supersymmetric Gauge Theories and Soliton Equations", Mod. Phys. Lett. A 11 (1996) 131, hep-th/9510183;

K. Takasaki, "Whitham Deformations and Tau Function in $\mathcal{N}=2 \mathrm{Su}$ persymmetric Gauge Theories", hep-th/9905224;

M. Marino, "The Uses of Whitham Hierarchies", hep-th/9905053.

[13] G. Bonelli and M. Matone, "Nonperturbative Relations in $\mathcal{N}=2 S U S Y$ Yang-Mills and WDVV Equation", Phys. Rev. Lett 77 (1996) 4712, hep-th/9605090;

A. Marshakov, A. Mironov and A. Morozov, "WDVV-Like Equations in $\mathcal{N}=2$ SUSY Yang-Mills Theory, Phys. Lett. B 389 (1996) 43, hepth/9607109.

[14] J. Edelstein, M. Marino and J. Mas, "Whitham Hierarchies, Instanton Corrections and Soft Supersymmetry Breakingin $\mathcal{N}=2 S U(N)$ SuperYang-Mills Theory", Nucl. Phys. B 541 (1999) 671, hepth/9805172;

J. Edelstein, M. Gomez-Reino and J. Mas, "Instanton Corrections in $\mathcal{N}=2$ Supersymmetric Theories with Classical Gauge Groups and Fundamental Matter Hypermultiplets", hep-th/9904087.

[15] J. Sonnenschein, S. Theisen and S. Yankielowicz, "On the Relation Between the Holomorphic Prepotential and the Quantum Moduli in SUSY Gauge Theories", Phys. Lett. B 367 (1996) 145, hep-th/9510129.

[16] E. D'Hoker, D.H. Phong and I.M. Krichever, "The Renormalization Group Equation in $\mathcal{N}=2$ Supersymmetric Gauge Theories", Nucl. Phys. B 494 (1997) 89, hep-th/9609145.

[17] Y. Ohta, "Prepotential of $\mathcal{N}=2$ SU(2) Yang-Mills Gauge Theory Coupled with a Massive Matter Multiplet", J. Math. Phys 37 (1996) 6074, hep-th/9604051; 
Y. Ohta, "Prepotentials of $\mathcal{N}=2$ SU(2) Yang-Mills Theories Coupled with Massive Matter Multiplets", J. Math. Phys 38 (1997) 682, hepth/9604059.

[18] H. Ewen and K. Forger, "Simple Calculation of Instanton Corrections in Massive $\mathcal{N}=2$ S SU(3) SYM", Int. J. Mod. Phys. A 12 (1997) 4725, hep-th/9610049;

H. Ewen, K. Forger and S. Theisen, "Prepotentials in $\mathcal{N}=2$ Supersymmetric SU(3) YM Theory with Massless Hypermultiplets", Nucl. Phys. B 485 (1997) 63, hep-th/9609062.

[19] E. D'Hoker, D.H. Phong and I.M. Krichever, "The Effective Prepotential of $\mathcal{N}=2$ Supersymmetric $S O\left(N_{c}\right)$ and $S p\left(N_{c}\right)$ Gauge Theories", Nucl. Phys. B 489 (1997) 211, hep-th/9609145.

[20] E. D'Hoker and D.H. Phong, "Strong Coupling Expansions of SU $(N)$ Seiberg-Witten Theory", Phys. Lett B 397 (1997) 94, hep-th/9701055.

[21] J. Edelstein and J. Mas, "Strong Coupling Expansion and SeibergWitten-Whitham Equations", Phys. Lett. B 452 (1999) 69, hepth/9901006.

[22] G. Chan, unpublished. 